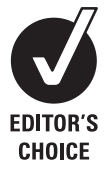

${ }^{1}$ Drug Health Services, Sydney South West Area Health Service, Sydney, Australia; ${ }^{2}$ Discipline of Addiction Medicine, Central Clinical School, University of Sydney, Sydney, Australia; ${ }^{3}$ Faculty of Medical Sciences, University of Groningen, Groningen, The Netherlands

Correspondence to: Dr Elizabeth Proude, Drug Health Services, Ground Floor, Page Building, Royal Prince Alfred Hospital, Missenden Rd, Camperdown NSW 2050, Australia; eproude@med.usyd.edu.au

Accepted 28 July 2008 Published Online First 15 August 2008

\title{
Nurse-provided screening and brief intervention for risky alcohol consumption by sexual health clinic patients
}

\author{
J Lane, ${ }^{1}$ E M Proude, ${ }^{2}$ K M Conigrave, ${ }^{1,2}$ J P de Boer, ${ }^{3}$ P S Haber ${ }^{1,2}$
}

\begin{abstract}
Objectives: Brief intervention for excessive alcohol consumption is effective yet not implemented widely. Alcohol misuse is implicated in unsafe sex and sexually transmitted infections and is common in clients of sexual health services. Our aims were to assess feasibility, acceptability and effectiveness of screening and brief intervention for risky alcohol consumption by a nurse in a sexual health clinic.
\end{abstract}

Methods: Patients completed the AUDIT questionnaire on handheld computers. Those scoring $\geqslant 8$ on AUDIT were asked to participate in the study and the 3 months' follow-up and were randomised to intervention or control groups. The Drink-less package (based on WHO validated methods) was used to implement the brief intervention by a trained registered nurse.

Results: Of 519 (87\%) who completed screening, 204 (39\%) scored $\geqslant 8$ on AUDIT (eligible), 184 agreed to follow-up and 133 completed it. At follow-up, both groups showed significant reductions in AUDIT scores. Mean scores decreased from 13.7 to 11.5 (control group) and 14.0 to 10.7 (intervention group); most (94\%) recalled the intervention and $62 \%$ reported reducing drinking compared with $47 \%$ of controls ( $p<0.001)$. The nurse screening and intervention process was reported acceptable by $74 \%$ of patients at follow-up and a majority (71\%) of staff.

Conclusions: Screening and brief intervention in a sexual health clinic for risky alcohol consumption is feasible, acceptable and effective in producing significant reductions in drinking as measured by AUDIT. Both intervention and control groups decreased consumption, suggesting that screening alone is sufficient to influence behaviour. Further study of brief intervention in this setting is appropriate.

Excessive consumption of alcohol is associated with a range of behavioural and health problems. In particular, alcohol is an important risk factor for unsafe sexual practices and is implicated in the spread of sexually transmitted infections (STIs). ${ }^{12}$ Several studies have been published about alcohol screening in sexual health clinics. The tools most often used are questionnaires-for example, AUDIT, ${ }^{13}$ CAGE and its variants, ${ }^{145}$ CRAFFT $^{1}$ and FAST ${ }^{6}$ - while others have used frequency and quantity questions, ${ }^{7}$ laboratory tests ${ }^{8}$ and blood alcohol reading. ${ }^{9}$ Brief advice has been shown to be effective in a variety of settings. ${ }^{10}$ Two studies reported attempts to give advice in a sexual health service. In one, $90 \%$ of the participants were willing to accept written material; however, less than a third agreed to accept an appointment with an alcohol health worker and only one person attended the appointment, leading to a disappointing result. ${ }^{11}$ This experience suggests that timely on-the-spot intervention is more likely to be deliverable. A pilot study reported a trial of the feasibility of screening and brief intervention for substance abuse in one clinic; however, the effect of the intervention was not measured and it was not the focus of the study. ${ }^{5}$

Our project tested the feasibility, acceptability and effectiveness of screening and brief intervention for risky alcohol consumption by sexual health clinic patients using a trained registered nurse. Effectiveness of the intervention was assessed by measuring recall of the intervention and any reported changes in drinking behaviour or reduction in levels of drinking by patients at 3 months after the index visit. Other aims were to assess the acceptability and efficiency of the screening and intervention process to practice staff.

\section{METHOD}

Sydney South West Area Health Service provides public sexual health clinics in two locations in central Sydney, Australia. Subjects were recruited at four to five clinic sessions per week, including one male-only clinic. Over a 6-month period, patients older than 16 years were asked by a research nurse to participate in a survey about alcohol while waiting to be seen by clinic staff (described as the index visit). Patients ineligible for screening were those with a language or literacy problem, mental health problem or who were too sick to participate.

The Alcohol Use Disorders Identification Test (AUDIT) questionnaire was programmed into a handheld computer with Pendragon Software 5.1 (Pendragon Software Corporation, Illinois, USA, 2005). Basic demographical information (age, gender) was also included. AUDIT is a validated 10-item instrument to detect hazardous and harmful drinking in ambulatory care settings. ${ }^{12}$ Three questions ask about quantity and frequency, three relate to possible symptoms of dependence and four ask about social and health problemswith a maximum possible score of 40 . A cut-off score of 8 on AUDIT is recommended, so that those who score between 8 and 15 are classed as risky drinkers, scores between 16 and 19 suggest hazardous use and scores of 20 and above clearly indicate further assessment for dependence. ${ }^{13}$ Question 3 is often used on its own (as AUDIT-3) to assess the frequency of drinking above recommended limits (that is, binge drinking).

All screening and intervention was conducted in privacy away from the waiting room. After 
completing the questions, the handheld computer was returned to the research nurse to check the score. Those scoring $<8$ were given feedback about their score and nationally recommended safe levels of drinking were reinforced. If the total score was $\geqslant 8$, or if the score for question 3 (AUDIT-3) was either 3 or 4 (that is, drinking more than six drinks on one occasion either daily or weekly), patients were asked to participate further and asked if they would be available for a follow-up telephone interview in 3 months' time. Those who provided written consent were assigned randomly to either the intervention or control group by pre-coded consent forms in sealed envelopes. This took about 5-10 minutes. The brief intervention (also 5-10 minutes), guided by the Drink-less handy card, ${ }^{14}$ was given to participants assigned to the intervention group. Two nurses had been previously separately trained in brief intervention for alcohol using the Drink-less package by one of the principal researchers and staff specialists in a one-on-one session lasting 1.5 hours. ${ }^{15}$

\section{Follow-up}

A structured telephone interview was developed for the 3 month follow-up, consisting of the AUDIT questionnaire (modified to reflect the shorter interval-3 months' timeframe as opposed to the original 12 months) and questions relating to any changes in alcohol consumption, having any recent treatment for alcohol problems, and recall and acceptability of being asked about alcohol and getting advice from a research nurse at their (index) visit to the clinic. At the end of the interview, all patients were offered feedback about the meaning of their AUDIT scores and were offered self-help materials (a Drink-less pocket guide); thus, ensuring that the control group patients were included. Follow-up data were directly entered into SPSS V.15 by the telephone interviewer who was blind to group status.

To assess the acceptability of the screening and intervention process to clinic staff, a seven-item self-administered survey was developed. Copies were mailed to the manager of the clinic after the final day of screening with cover letters and reply paid envelopes for return.

\section{Statistical analysis}

Frequencies were calculated for all variables. $\chi^{2}$ analyses were also carried out to compare risky and high-risk drinkers, defined by their AUDIT scores (8-12 for risky, $>13$ for high-risk) from baseline to follow-up, for both groups. These cut-off scores were chosen because they follow those given in the Drink-less intervention package. ${ }^{15}$ Paired samples $t$ tests were also performed to test for levels of significance in the differences in mean AUDIT scores, question 3 for episodic heavy drinking (AUDIT-3) and for AUDIT-C (the first 3 questions of AUDIT), from baseline to follow-up for both groups.

\section{RESULTS}

Altogether, 599 patients were approached to participate in the study and 519 (87\%) agreed to initial screening. Of the 511 patients who provided demographical data, 377 (74\%) were male and $134(26 \%)$ were female. The mean age was 34 years (range 16-81; SD 10.7). Of the 80 people who declined or were ineligible, 54 (66\%) were male and 26 (34\%) female.

Altogether, $40 \%$ of patients had a total AUDIT score $\geqslant 8: 22 \%$ of all patients screened scored between 8 and 12 suggesting hazardous drinking, $12 \%$ had scores between 13 and 19 , and $6 \%$ scored $\geqslant 20$. Therefore, $18 \%$ were drinking at levels likely to be harmful or dependent. ${ }^{14}$ The AUDIT-C was significantly correlated with the total AUDIT score $(p<0.01, r=0.814)$.

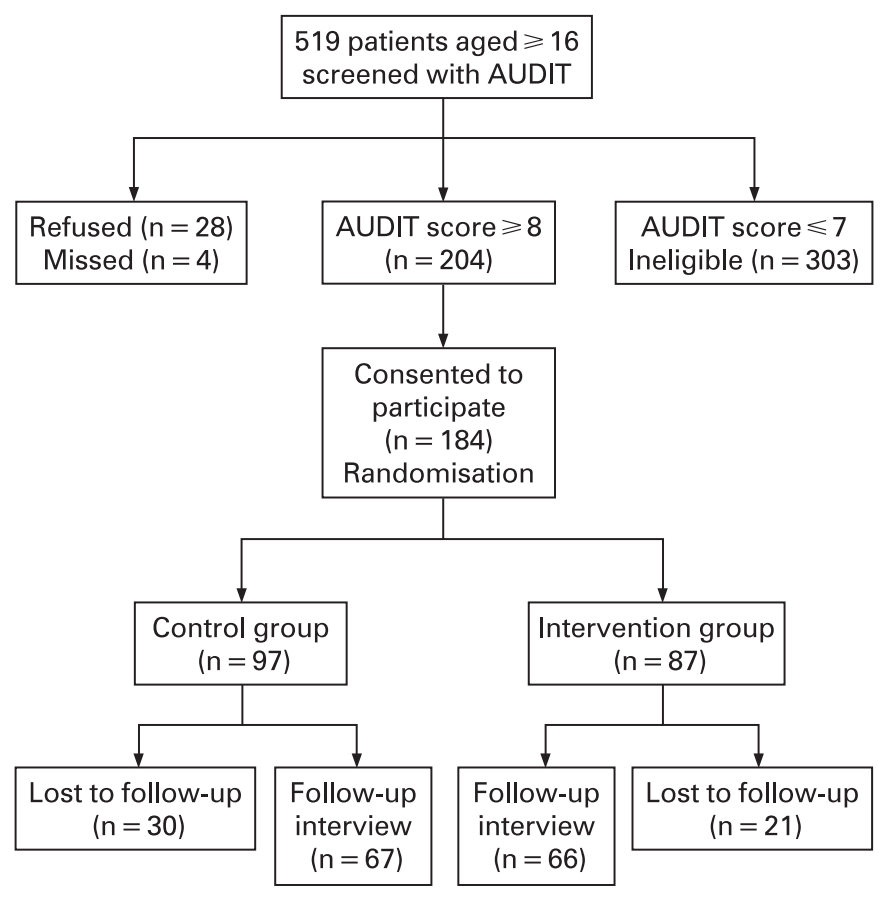

Figure 1 Flowchart of patients participating.

The total eligible for inclusion in the study was 204 patients; 28 patients refused to participate further and 4 patients were inadvertently not included, leaving 184 in the study population (87 patients in the intervention group and 97 patients in the control group) (fig 1). There were no differences in baseline scores between the control and intervention groups or between males and females.

\section{Follow-up}

Patients scoring $\geqslant 20$ or more on AUDIT, and therefore at a high risk of dependence, were less likely to agree to participate in follow-up ( $p=0.093$, data not shown). Altogether, 133 patients (67 (69\%) of controls and 66 (76\%) of the intervention group) were followed up at a 3-month interval. Those lost to follow-up did not differ in age or gender from those re-interviewed. Again, however, patients in the highest risk category were significantly more likely to be uncontactable (table 1).

Of all those followed, 41 (31\%) of patients reduced their AUDIT scores to a level where they were no longer categorised as drinking at either hazardous or harmful levels. The proportions of patients drinking at any risk level were correspondingly reduced for both the control and intervention groups (table 2). The proportion of people drinking more than six drinks daily, almost daily or weekly, as measured by AUDIT3 , was reduced by $12 \%$ in the intervention group (from $51 \%$ to $39 \%)$. The control group remained stable at $46 \%$. AUDIT scores and AUDIT-C scores (questions 1-3) were all normally distributed for both groups at baseline. Mean AUDIT scores

Table 1 Comparison of baseline AUDIT scores and follow-up status

\begin{tabular}{llll}
\hline $\begin{array}{l}\text { AUDIT score at } \\
\text { baseline }\end{array}$ & $\begin{array}{l}\text { Lost to follow-up } \\
(\mathbf{n}=\mathbf{5 1}), \mathbf{n}(\%)\end{array}$ & $\begin{array}{l}\text { Completed follow-up } \\
(\mathbf{n}=\mathbf{1 3 3}), \mathbf{n}(\%)\end{array}$ & Total $(\mathbf{n}=\mathbf{1 8 4}), \mathbf{n}(\%)$ \\
\hline $8-12$ & $32(63)$ & $67(50)$ & $99(54)$ \\
$13-19$ & $7(14)$ & $51(38)$ & $58(31)$ \\
$\geqslant 20$ & $12(23)$ & $15(11)$ & $27(15)$ \\
& & & \\
& & & \\
\hline
\end{tabular}


Table 2 Comparison of AUDIT scores and AUDIT-3 high scores at baseline and follow-up for both groups (followed-up patients only)

\begin{tabular}{|c|c|c|c|c|}
\hline & \multicolumn{2}{|l|}{ Baseline } & \multicolumn{2}{|l|}{ Follow-up } \\
\hline & $\begin{array}{l}\text { Control } \\
(n=67) \\
n(\%)\end{array}$ & $\begin{array}{l}\text { Intervention } \\
(\mathrm{n}=66) \\
\mathrm{n}(\%)\end{array}$ & $\begin{array}{l}\text { Control } \\
(n=67) \\
n(\%)\end{array}$ & $\begin{array}{l}\text { Intervention } \\
(\mathrm{n}=66) \\
\mathrm{n}(\%)\end{array}$ \\
\hline \multicolumn{5}{|l|}{ AUDIT score } \\
\hline $0-7$ & $0(0)$ & $0(0)$ & $20(30)$ & $21(32)$ \\
\hline \multicolumn{5}{|l|}{ Low risk } \\
\hline $8-12$ & $34(51)$ & $33(50)$ & $26(39)$ & $26(39)$ \\
\hline \multicolumn{5}{|l|}{ Hazardous level } \\
\hline $13-19$ & $27(40)$ & $24(36)$ & $12(18)$ & $15(23)$ \\
\hline \multicolumn{5}{|l|}{ Harmful level } \\
\hline $\begin{array}{l}\geqslant 20 \text { Risk of } \\
\text { dependence }\end{array}$ & $6(9)$ & $9(14)$ & $9(13)$ & $4(6)$ \\
\hline AUDIT-3 score $\geqslant 3$ & $30(46)$ & $32(51)$ & $31(46)$ & $26(39)$ \\
\hline
\end{tabular}

decreased from 13.7 to 11.5 (difference of 2.2) for the control group and from 14.0 to 10.7 (difference of 3.3) for the intervention group. Both groups showed significant changes in 10-item AUDIT scores from baseline to follow-up, but the amount of change did not differ significantly between the groups. The intervention group showed a significant reduction in their AUDIT-C score and a slight lowering of their AUDIT-3 score (table 3).

All patients were asked whether their general practitioner had ever asked them about alcohol, whether they had seen any other health professional about their drinking or had any treatment for alcohol problems in the past 3 months. When asked if they remembered any advice about alcohol at the index visit, $94 \%$ of the intervention group recalled the advice, $80 \%$ thought the advice had been acceptable and $75 \%$ of all patients thought it would be acceptable from a nurse (table 4). More of the intervention group found it acceptable to receive advice about alcohol on a routine visit compared with control group.

Patients were also asked about any change in drinking habits in the past 3 months. Altogether, $62 \%$ of the intervention group reported that they had reduced their drinking, whereas only $47 \%$ of controls had done so, showing a tendency towards significance $(p=0.09)$.

\section{Acceptability and efficiency of the process to clinic staff}

All clinic staff completed the brief survey. Five out of seven found that the presence of the nurse made little impact on the routine of the clinic; two found it inconvenient because of shortage of space. The concern about space was considered valid as we had to restrict screening to the sessions when a room was free. Staff felt it useful to have a nurse screen patients for excessive alcohol consumption; all clinicians thought it was important to know about a patient's alcohol use and that it could be incorporated into their role to screen and give advice if time allowed. They nominated doctors, nurses and counsellors all as appropriate people to provide the service.

\section{DISCUSSION}

Our study reinforces previous findings that patients of sexual health clinics have higher levels of risky alcohol consumption than those in the general population or patients in other healthcare settings. Over $40 \%$ of patients we screened were drinking at hazardous or harmful levels compared with an average of $30 \%$ in the general population -with more than $20 \%$ of patients drinking more than six standard drinks at least once a week. This is consistent with other studies conducted in
Table 3 Paired samples $t$ tests for differences between baseline and follow-up AUDIT scores, AUDIT-C scores and AUDIT-3 scores, by group

\begin{tabular}{llll}
\hline & $\begin{array}{l}\text { Downward change in } \\
\text { mean (95\% CI) }\end{array}$ & t test & p Value \\
\hline $\begin{array}{l}\text { Intervention } \\
\text { AUDIT score base to } \\
\text { follow-up }\end{array}$ & $3.3(2.1$ to 4.8$)$ & 5.1 & $\mathrm{p}<0.001^{*}$ \\
$\begin{array}{l}\text { AUDIT-C score base to } \\
\text { follow-up }\end{array}$ & $0.8(0.18$ to 1.4$)$ & 2.6 & $\mathrm{p}=0.01^{*}$ \\
$\begin{array}{l}\text { AUDIT-3 base to } \\
\text { follow-up }\end{array}$ & $0.1(-0.02$ to 0.02$)$ & 1.7 & $\mathrm{p}=0.09$ \\
Control \\
$\begin{array}{l}\text { AUDIT score base to } \\
\text { follow-up }\end{array}$ & $2.2(1.06$ to 3.4$)$ & & \\
$\begin{array}{l}\text { AUDIT-C score base to } \\
\text { follow-up }\end{array}$ & $0.4(-0.06$ to 0.8$)$ & 3.3 & $\mathrm{p}<0.001^{*}$ \\
$\begin{array}{l}\text { AUDIT-3 base to } \\
\text { follow-up }\end{array}$ & $0.0(-0.14$ to 0.14$)$ & 1.7 & $\mathrm{p}=0.08$ \\
\hline
\end{tabular}

*Significant.

sexual health clinics where the prevalence of heavy drinking ranged from approximately $30 \%{ }^{411}$ to $65 \% .^{6}$

The screening and advice session was innovative in using handheld computers and patients were extremely interested in their results. Both intervention and control patients significantly decreased their AUDIT score after 3 months and the intervention group also significantly reduced their AUDIT-C score. Comments from respondents included "this was a wake-up

Table 4 General practitioners' questions about alcohol, and recall and acceptability of the nurse intervention

\begin{tabular}{lll}
\hline & Intervention & \\
$(n=66)$ & Control $(n=66)$ \\
$n(\%)$ & $n(\%)$
\end{tabular}

Has your current (or last) GP ever asked you about alcohol?

$\begin{array}{lcc}\text { Yes } & 19(29) & 28(41) \\ \text { No } & 42(64) & 35(54) \\ \text { Can't remember } & 5(7) & 2(3)\end{array}$

NS

Have you seen any other health professional about alcohol in the last 3 months?

$\begin{array}{lcc}\text { Yes } & 6(9) & 5(8) \\ \text { No } & 60(91) & 61(92) \\ & & \text { NS }\end{array}$

Have you received any treatment for alcohol problems in last 3 months?

$\begin{array}{lcc}\text { Yes } & 2(3) & 2(3) \\ \text { No } & 64(97) & 64(97) \\ & & \text { NS }\end{array}$

Do you remember getting advice about alcohol at the clinic 3 months ago?

(ie, at the index visit)

Yes

No/don't know

$62(94) \quad 13(20)$

$4(6) \quad 53(80)$

$\chi^{2}=p<0.001$

Do you think it acceptable to get any advice about alcohol on a routine visit to the clinic? Yes

$53(80)$

$46(70)$

Unsure/No

$13(20)$

20 (30)

$\chi^{2}=p=0.159$

Would it be acceptable to get this advice about alcohol from a nurse?
Yes
$49(74)$
$50(76)$
Unsure/No
17 (26)
16 (24)

NS 


\section{Key messages}

- Patients attending sexual health clinics have higher levels of risky alcohol consumption compared with patients in other clinical settings.

- Screening for risky alcohol use can be incorporated into a routine clinic visit.

- Brief advice (5-10 minutes) on alcohol consumption should be given there and then.

- Advice on alcohol is acceptable to the majority of patients.

call for me". Both groups reduced their drinking, suggesting that screening alone is sufficient to produce an effect on alcohol consumption.

The extreme mobility of the patient population presented a challenge to successful follow-up; however, this was partly overcome by the use of follow-up phone calls to mobile telephones made out of business hours. Constraints on space within the clinics were a problem and we were only able to screen patients on selected days when room was available. This caused the screening and intervention process to lengthen in order to recruit sufficient patients to provide sufficient power for the study.

Limitations of the study included the reluctance of those with higher baseline AUDIT scores to participate. This meant that a group with the potential for larger changes in alcohol consumption did not participate. This reflects a characteristic of alcohol use disorders-that is, a greater dependence on alcohol as the disorder progresses. The use of AUDIT as a tool to examine changes in drinking over 3 months presented both advantages and disadvantages for this study. A more detailed measure of quantity and frequency may have revealed significant differences in alcohol consumption at follow-up. However, AUDIT was selected as a simple and validated measure that could easily be done at both baseline and follow-up in a client group attending a busy clinic with limited time to participate in a study of this type.

Excessive alcohol consumption is associated with myriad adverse effects and accounts for approximately $4 \%$ of total morbidity in Australia. ${ }^{16}$ Early intervention is one of the most promising approaches to address alcohol problems, yet it is not implemented in many settings where it might be particularly effective. Incorporating a brief screening instrument (for example, AUDIT-C) would add no more than 1 minute to a consultation; a brief intervention could be given in 5 minutes. Economic costs would be more than offset by the benefit accruing from reduced future demand on clinical services for alcohol-related conditions. ${ }^{17}$ This study has shown that a nurse with a modest level of training in this intervention can be successfully introduced into a clinic with a relatively high proportion of patients who experience alcohol problems. Space and time pressures may have limited our implementation. It is also recognised that in order to produce an intervention effect that persists beyond a 3 month interval, reinforcement of advice at subsequent visits may be necessary. Nevertheless, integration of alcohol screening into the core role of the sexual health clinic nursing staff would appear to be an appropriate strategy to explore for the further implementation of brief intervention for alcohol use disorders in this setting.

Acknowledgements: Thanks to Dr Catherine O'Connor, Dr Michael Moore and Darren Smyth of Sydney South West Area Health Service; and to Loretta Healey and the staff and clients of both Livingstone Road and The Sanctuary Clinics. Thanks also to Dr Kirsten Morley for statistical advice.

Funding: The Drug and Alcohol Council, New South Wales Health Department provided funding for the study. The funding body had no part in the conduct or design of the design.

Competing interests: None.

Ethics approval: Ethics approval for the study was obtained from the Ethics Review Committee of Sydney South West Area Health Service (RPA zone).

Contributors: $\mathrm{EP}, \mathrm{KC}$ and $\mathrm{PH}$ obtained the funding for the project. JdB took a substantial role in the follow-up and wrote a full report on which this paper is based. $\mathrm{JL}$ carried out the majority of the field work and wrote the first draft of the paper. EP was responsible for the conduct of the study, carried out a full analysis of results, wrote the second draft and completed subsequent revisions of the paper. $\mathrm{KC}$ and $\mathrm{PH}$ supervised the project, provided statistical advice, trained the research nurses and reviewed all versions of the paper prior to submission. All authors approved the final version.

\section{REFERENCES}

1. Cook RL, Chung T, Kelly TM, et al. Alcohol screening in young persons attending a sexually transmitted disease clinic. Comparison of AUDIT, CRAFFT, and CAGE instruments. J Gen Intern Med 2005;20:1-6.

2. Catalan J, Day A, Gallwey J. Alcohol misuse in patients attending a genitourinary clinic. Alcohol 1988;23:421-8.

3. Kalichman SC, Cain D, Zweben A, et al. Sensation seeking, alcohol use and sexual risk behaviors among men receiving services at a clinic for sexually transmitted infections. J Stud Alcohol 2003;64:564-9.

4. Appel P, Piculell R, Jansky $\mathrm{H}$, et al. Assessing alcohol and other drug problems (AOD) among sexually transmitted disease (STD) clinic patients with a modified CAGE-A: implications for AOD intervention services and STD prevention. Am J Drug Alcohol Abuse 2006;32:225-36.

5. Yu J, Appel PW, Warren B, et al. Substance abuse intervention services in public sexually transmitted disease clinics; a pilot experience. J Subst Abuse Treat 2008;34:356-62.

6. Standerwick K, Davies C, Tucker L, et al. Binge drinking, sexual behaviour and sexually transmitted infection in the UK. Int J STD AIDS 2007;18:810-13.

7. Zenilman JM, Hook EW 3rd, Shepherd M, et al. Alcohol and other substance use in STD clinic patients: relationships with STDs and prevalent HIV infection. Sex Transm Dis 1994;21:220-5.

8. Liebschutz JM, Finley EP, Braslins PG, et al. Screening for sexually transmitted infections in substance abuse treatment programs. Drug Alcohol Depend 2003;70:93-9.

9. Schofield C, Wilson E, Patel A, et al. Blood alcohol concentrations in patients attending special clinics in Glasgow. British Venereal Disease 1975;51:34-344.

10. Moyer A, Finney J. Brief intervention for alcohol problems. Alcohol Res Health 2005;28:44-50

11. Crawford MJ, Lowe PC, Greene L, et al. The prevalence of excessive alcohol consumption and the acceptability of brief advice in a sexual health clinic: cross sectional survey. Sex Transm Infect 2004;80:416-7.

12. Saunders JB, Aasland OG, Babor TF, et al. Development of the Alcohol Use Disorders Identification Test (AUDIT): WHO collaborative project on early detection of persons with harmful alcohol consumption - II. Addiction 1993;88:791-804.

13. Babor T, Higgins-Biddle J, Saunders J, et al. The Alcohol Use Disorders Identification Test: Guidelines for use in primary care. Geneva: World Health Organization, 2001.

14. Proude E, Saunders J, Conigrave K, et al. The Drink-less Program 2005.

15. Proude EM, Conigrave KM, Haber PS. Effectiveness of skills-based training using the Drink-less package to increase family practitioner confidence in intervening for alcohol use disorders. BMC Med Edu 2006;6:8.

16. National Alcohol Strategy: a plan for action 2001 to 2004. Canberra: Commonwealth of Australia, 2001.

17. Solberg LI, Maciosek MV, Edwards NM. Primary care intervention to reduce alcohol misuse: ranking its health impact and cost effectiveness. Am J Prev Med 2008:34:143-52. 